\title{
Inovação e geração de conhecimento científico e tecnológico no Brasil: uma análise dos dados de cooperação da Pintec segundo porte e origem de capital*
}

\author{
Carlos Pinkusfeld Bastos** \\ Jorge Britto ${ }^{* * *}$
}

Recebido: 12/01/2016 Versão Revisada (entregue): 19/02/2016 Aprovado: 06/04/2016

\section{RESUMO}

Este artigo tem como objetivo central a avaliação quantitativa das relações cooperativas desenvolvidas entre a estrutura produtiva brasileira envolvida com atividade inovativa, universidades e centros tecnológicos. A análise aborda essas relaçôes tanto em termos de sua evolução no tempo como segundo características específicas das empresas, tais como porte e origem de capital. O trabalho empírico utiliza tabulaçôes especiais da Pesquisa de Inovação (Pintec), realizada pelo Instituto Brasileiro de Geografia e Estatística (IBGE). As evidências apresentadas reforçam a constatação de que esse padrão de interação é bastante complexo e multifacetado, confirmando que variáveis como porte e origem de capital têm impacto relevante sobre a intensidade das relações de cooperação. $\mathrm{Na}$ análise temporal constatou-se uma perceptível melhoria dos indicadores que apontam para intensificação de práticas cooperativas na última Pintec (2011). Ainda que outros fatores, tanto micro como macroeconômico, conjunturais devam ser considerados, sugere-se que essa tendência pode representar um vetor positivo no que se refere às perspectivas de desenvolvimento da indústria brasileira.

\footnotetext{
* Este texto foi elaborado com base em estudo realizado para o Seminário "14 anos de Pintec - Qualidade do Investimento no Brasil e Desafio da Política de Inovação 2015-2018”, organizado pela Finep-PNUD.

Os autores agradecem os comentários e sugestôes de dois pareceristas anônimos. Este artigo contou com o apoio financeiro da Finep, que também forneceu os dados aqui utilizados.

** Universidade Federal do Rio de Janeiro (UFRJ), Rio de Janeiro (RJ), Brasil. E-mail: pinkusfeld@gmail.com

*** Universidade Federal Fluminense (UFF), Niterói (RJ), Brasil. E-mail: britto.jorge@gmail.com.
} 
PalaVRaS-CHAVE | Cooperação Universidade-Indústria; Pintec-Cooperação; Sistema Nacional de Inovação; Interação e Cooperação

Códigos JEL | L52; L24; O14; O25; O32

\section{Innovation and scientific and technological knowledge in Brazil: as analysis of PINTEC's cooperation data according to size and capital origin}

\section{ABSTRACT}

The main objective of this article is a quantitative evaluation of cooperative relations established between the Brazilian productive structure involved in innovative activities, universities and technology centers. The analysis addresses these relations both in terms of its evolution in time as well as according to specific business characteristics, such as size and capital origin. The empirical work is based on special tabulations from PINTEC-IBGE, the Brazilian Innovation Survey. The evidence reinforces the fact that this pattern of interaction is very complex and multifaceted confirming that variables such as size and source of capital have a significant impact on the intensity of cooperative relations. In temporal analysis we found a noticeable improvement of the intensification of cooperative practices in the last PINTEC (2011). Although other factors related to both micro and macroeconomic conjuncture should be considered, it is suggested that this trend may represent a positive vector in relation to the Brazilian industry development prospects.

KEYWORDS | University-Industry Cooperation; Cooperation-Brazilian Innovation Survey; National Innovation System; Interaction and Cooperation

JEL-CODES | L52; L24; O14; O25; O32 


\section{Introdução}

A construção de um Sistema Nacional de Inovação (SNI) é o passo central para o desenvolvimento endógeno de tecnologia e, consequentemente, de uma trajetória de desenvolvimento econômico sólido e sustentável. Um dos elementos fundamentais para tal construção é a criação de relações de cooperação entre empresas, centros tecnológicos e universidades. O presente trabalho discute as relaçôes cooperativas estabelecidas entre empresas inovativas e centros de produção científico-tecnológica no caso brasileiro. Este recorte teórico se organiza em torno de algumas hipóteses centrais. A primeira refere-se à existência de uma correlação positiva entre o estreitamento e ampliação das relações de cooperação e o desempenho inovativo das empresas. Por outro lado, a segunda entende que a construção de relaçôes cooperativas depende de características intrínsecas das distintas empresas, tanto em termos de dimensão quanto de propriedade de capital. Finalmente, o terceiro vetor central desta relação está nas iniciativas de políticas públicas que podem promover tais relaçôes. Com esse intuito, o artigo recorre à utilização de tabulações especiais da Pesquisa de Inovação (Pintec), realizada pelo Instituto Brasileiro de Geografia e Estatística (IBGE), a partir das quais a intensidade e o desempenho inovativo das empresas são associados à cooperação com universidades, institutos de pesquisa e com centros de capacitação profissional e assistência técnica. Inicialmente apresenta-se uma breve fundamentação teórica da importância das relações cooperativas para o processo de inovação tecnológica, seguida de uma revisão de trabalhos publicados sobre tema no Brasil que utilizam diferentes metodologias, incluindo evidências de bases de informações complementares à Pintec. Em sequência, busca-se o exame empírico dos eixos teóricos apontados anteriormente, por meio da exploração dos dados originais da Pintec, a partir dos quais as evidências da cooperação entre empresas e infraestrutura de C\&T são analisadas segundo o porte e a origem do capital das empresas. Por fim, uma seção conclusiva procura contextualizar os resultados apresentados com o comportamento da economia brasileira e com as açóes da política de C,T\&I.

\section{Cooperação universidade-indústria no âmbito dos Sistemas Nacionais de Inovação}

A interação da produção científica e tecnológica constitui um aspecto crucial para o a consolidação de Sistemas Nacionais de Inovação (SNIs), envolvendo tanto a 
absorção de conhecimentos produzidos em universidades e institutos de pesquisa pelo setor produtivo, conforme comprovam estudos realizados por Lundvall (1992, 2002), Klevorick et al. (1995), Narin et al. (1997) e Cohen et al. (2002), como, por outro lado, a capacidade do conhecimento tecnológico gerado pelo setor produtivo em fornecer questôes para a elaboração científica, conforme descrito por Rosenberg (1982). Como reflexo desse processo, consolidam-se fluxos bidirecionais e mutuamente dinâmicos entre essas duas instituições dos sistemas de inovação, de acordo com o discutido nas análises de Brascomb et al. (1999) e Mowery et al. (2004). A importância dessas articulações para acelerar processos de catching-up é reconhecida e abordada empiricamente na literatura (MAZZOLENI, 2008; MAZZOLENI; NELSON, 2007; ALBUQUERQUE et al., 2015).

Os modelos institucionais de interação incluem um amplo conjunto de possibilidades, que se estruturam em função do desenvolvimento atingido em determinadas áreas científicas e das características dos setores produtivos impactados. Diferentes investigaçôes têm abordado os motivos para a cooperação em P\&D e inovação a partir de distintas perspectivas (ARCHIBUGI; IAMMARINO, 2002; TETHER, 2002). Os modelos organizacionais mobilizados para viabilizar a aproximação entre o setor produtivo e a infraestrutura de C\&T podem ser extremamente variados (BONACCORSI; PICCALUGA, 1994), incluindo joint-ventures, incubadoras de empresas, parques tecnológicos, consórcios de $\mathrm{P} \& \mathrm{D}$, centros de transferência de tecnologia, centros de pesquisa cooperativa, instituições-ponte dedicadas ao gerenciamento de contratos de pesquisa, spin-offs de empresas de base tecnológica e múltiplas formas de alianças entre indústria, governo e universidades. Para alguns autores, a sofisticação dessas formas de articulação evidenciaria a paulatina consolidação de um novo "modo geral" de articulação entre as duas esferas (e o setor governamental) expresso na ideia de uma "hélice tríplice", indutora da intensificação de processos inovativos (ETZKOWITZ; LEYDERSDORFF, 2000; ETZKOWITZ, 2013). A partir da constatação da relevância das articulaçôes entre instituições de pesquisa e o setor produtivo nas trajetórias de desenvolvimento dos distintos Sistemas Nacionais de Inovaçôes (SNIs), Suzigan e Albuquerque (2008) e Rapini (2007) identificam algumas funções fundamentais da universidade no âmbito desses sistemas: formação de pessoal em geral; formação de pessoal capacitado para fundar novas empresas, especialmente em novas áreas tecnológicas ("spin-offs"); fonte de conhecimento de caráter mais geral necessário para as atividades de pesquisa básica e fonte de conhecimento especializado relacionado à área tecnológica da firma; geração de conhecimento na forma de "spillovers" (utilizados por empresas e outros agentes com capacidade 
de absorção previamente construída); estabelecimento de interações diretas com empresas estabelecidas, favorecendo processos inovativos adaptados às exigências do setor empresarial; e estabelecimento de canais para absorção de conhecimento gerado nos centros mais avançados. A relevância dessas funçōes variaria de acordo com as especificidades institucionais, os padrões de especialização nos campos científico e produtivo e as trajetórias evolutivas dos diferentes Sistemas Nacionais de Inovações.

Diversas análises avançam na discussão dos determinantes da intensidade e dos impactos da cooperação universidade-indústria, a partir de abordagens e metodologias microfundamentadas. Quanto à transmissão de conhecimento, observa-se que tanto trabalhos pioneiros (KLEVORICK, et al., 1995) quanto mais recentes (BEKKERS; BODAS-FREITAS, 2008) colocam a mobilidade de recursos humanos entre universidades e empresas como uma das suas formas mais importantes. As universidades oferecem tanto o conhecimento mais geral por meio de recém-graduados quanto o conhecimento mais específico retido por pesquisadores de ponta (KLEVORICK et al., 1995). A publicação de resultados de $\mathrm{P} \& \mathrm{D}$ por artigos acadêmicos também é uma forte fonte de transmissão de conhecimento, sendo, porém, menos comum, dado que as firmas especificam tais termos em contratos (PERKMANN; WALSH, 2008). Os trabalhos de Fuentes e Dutrénit (2012) e Arza (2010) apontam que os canais de interação encontram-se separados em certos grupos, dependendo do seu grau de formalização, do seu grau de interação, da direção dos fluxos de conhecimento e da natureza dos resultados aplicados.

Ao se analisarem diversas experiências nacionais, verifica-se uma grande variedade das formas de contribuição das instituições de C\&T para o desenvolvimento industrial (RAPINI; CASSIOLATO; BITTENCOURT, 2007; ALBUQUERQUE et al., 2015). No caso específico dos países em desenvolvimento, destaca-se o papel da ciência na contribuição para o processo de catching-up, na medida em que a infraestrutura científica atua como um "instrumento de focalização" e como uma "antena" para identificar oportunidades tecnológicas, permitindo vincular o país aos fluxos científicos e tecnológicos internacionais, apoiando o seu desenvolvimento industrial e atuando como fonte para soluçôes criativas que dificilmente seriam obtidas fora do país (como, por exemplo, no caso de vacinas contra doenças tropicais). Além disso, a interação entre a geração de conhecimento científico e tecnológico permite o desenvolvimento da capacidade de absorção de novos conhecimentos por parte da firma inovativa, que desenvolve P\&D em colaboração com instituições científicas.

No caso latino-americano, em função do baixo nível de P\&D interno realizado pelas empresas locais, Arocena e Sutz (2003) encontram evidências de que as arti- 
culações universidade-empresa tendem a ficar circunscritas à prestação de serviços rotineiros e a atividades de consultoria técnica, raramente envolvendo atividades de pesquisa de caráter mais experimental. Na mesma linha, Dutrenit e Arza (2010) argumentam que, ao contrário do que ocorre nos países desenvolvidos, as firmas nesses países procuram as universidades mais para resolver problemas de curto prazo do que para buscar alternativas de inovação de longo prazo. Isso pode ser explicado pelo excesso de empresas estrangeiras atuando em setores dinâmicos na região, conforme sugerido por Schartinger et al. (2002). Entretanto, os grupos de pesquisa na América Latina também assumem papel importante quando operam como antena para captar o conhecimento gerado fora do país e traduzi-lo para as necessidades locais, o que viabilizaria o catching-up realizado em alguns setores dessas economias. Nesse sentido, Rapin (2007) e Rapini et al. (2013) observam na região uma mudança de postura das universidades e instituições de pesquisa, visando acelerar investigações orientadas para a busca de avanços que tenham maior relevância para a sociedade, por meio de pesquisas mais densas que possibilitem uma comercialização efetiva dos resultados gerados.

\section{A interação universidade-empresa no Brasil: evidências gerais}

Desde o final dos anos 1990, observa-se uma mudança da política de C,T\&I no Brasil incorporando a busca de uma maior integração com a política industrial. Neste contexto, o financiamento público passa a ser encaminhado de forma mais direta para empresas inovadoras e, paulatinamente, intensificam-se os estímulos à formação de redes de cooperação entre empresas e centros de pesquisa e universidades. Esta evolução das políticas públicas reflete-se tanto na ampliação de recursos quanto no escopo dos programas, que passam a incorporar um conceito amplo de inovação. Tal conceito está articulado a uma visão sistêmica do Sistema Nacional de Inovação brasileiro, que ressalta a necessidade de adequação das condições de financiamento às empresas, em conjunto com a importância da estruturação de redes de instituições e da formação e recursos humanos qualificados (BRITTO; STALLIVIERI, 2011).

Dentre os instrumentos que surgiram ou foram fortalecidos na última década, de forma a operacionalizar estas políticas, destacam-se a criação dos Fundos Setoriais, a Lei de Inovação, a Lei do Bem, os programas de financiamentos e subsídios econômicos às empresas implementados pela Finep e o BNDES e, mais recentemente, o arcabouço institucional da Estratégia Nacional de Ciência, Tecnologia Inovação (ENCTI - 2012/2015), que sucedeu o Plano de Ação de Ciência, Tecnologia e 
Inovação para o Desenvolvimento Nacional (PACTI 2007-2010). A ENCTI aponta, expressamente, o investimento em C,T\&I como eixo estruturante do desenvolvimento do país e ratifica o papel da inovação em seu esforço de desenvolvimento sustentável e na consolidação de um novo padrão de inserção internacional brasileiro. Ao longo dessa evolução, ampliou-se e sofisticou-se o arsenal de incentivos ao fortalecimento das conexôes e cooperações entre empresas, institutos de pesquisa e universidades.

Estas mudanças têm ocorrido num contexto marcado por algumas características particulares do sistema de inovação brasileiro, discutidas por Suzigan e Albuquerque (2011). Uma dessas características é a existência de instituições de pesquisas firmemente estabelecidas, mas que ainda não conseguem mobilizar contingentes de pesquisadores, cientistas e engenheiros semelhantes aos dos países mais desenvolvidos. Da mesma forma, as firmas ainda têm um envolvimento relativamente restrito em atividades inovativas, o que restringe a dinâmica interativa entre empresas e universidades, a partir da qual se constituem circuitos de retroalimentação positiva entre as dimensões científica e tecnológica. Assim, a despeito da existência de interações pontuais importantes, muitas das quais estão por detrás de experiências bem-sucedidas em termos de ganhos de competitividade internacional, esse não é um padrão generalizado que perpassa o conjunto das atividades econômicas.

Em face das evidências que apontam para o caráter limitado dessas interações no caso brasileiro (VELHO; SAENZ, 2002; SEGATTO; SBRAGIA, 2003; DAGNINO, 2004; BERNARDES; ALBUQUERQUE, 2003; ALBUQUERQUE et al., 2005; RIGHI; RAPINI, 2007), é importante identificar quais as especificidades deste padrão de interação. A infraestrutura de C\&T brasileira é complexa e envolve um elevado número de instituições, incluindo universidades públicas e privadas e centros de pesquisa, sendo observada grande heterogeneidade nos padrões de relacionamentos com o setor produtivo, conforme apontam estudos mais recentes sobre o tema (FREITAS et al., 2013; FERNANDES et al., 2010; SUZIGAN; ALBUQUERQUE; CARIO, 2011; RIGHI, 2009; RAPINI et al., 2013; AZEVEDO; CARIO; MELO, 2015; PINHO; TORKOMIAN; SANTOS, 2015).

Em função da heterogeneidade dos padrões colaborativos observados no caso brasileiro, há evidências de que, diferentemente do proposto por Arocena e Sutz (2003), a interação com o setor produtivo não se dá apenas por meio de consultorias, da contratação de recém-graduados e das demais atividades tradicionais, incluindo também um número significativo de instituições que atuam em campos de pesquisa relevantes para o desenvolvimento de novas tecnologias ou até mesmo em pesquisas de fronteira em setores de alta tecnologia (RAPINI et al., 2013; GONÇALO; 
ZANLUCHI, 2011; PARANHOS; HASENCLEVER, 2009). Percebe-se, assim, que as universidades/institutos de pesquisa cumprem um duplo papel no sistema de inovação brasileiro. Em particular, as universidades funcionam como uma antena que capta, codifica e transmite o conhecimento gerado nos países centrais, de modo a aplicá-lo às necessidades brasileiras (RAPINI, 2007). Além disso, as universidades são apontadas pelas empresas como particularmente importantes para a sugestão de novos projetos de pesquisa no Brasil, aspecto que se distingue de resultados encontrados para países desenvolvidos, nos quais a definição desses projetos usualmente decorre da identificação prévia de áreas estratégicas de interesse pelo setor empresarial (RAPINI et al., 2013). Desse modo, o papel da infraestrutura de C\&T para o fortalecimento do Sistema Nacional de Inovação tende a ser reforçado.

$\mathrm{Na}$ discussão dos determinantes e impactos dessas interações no caso brasileiro, vários trabalhos recentes têm procurado identificar características das firmas que levam ao aumento das interações, como o porte (PÓVOA; MONSUETO, 2011), se a empresa é parte de uma grande corporação (FUENTES; DUTRÉNIT, 2012), o setor em que elas se inserem (ALBUQUERQUE; SILVA; PÓVOA, 2005; ALBUQUERQUE et al., 2008; BRITTO; OLIVEIRA, 2011), a intensidade tecnológica desses setores (PINHO, 2011; BRITTO; DEL VECCHIO, 2014) e o fato de uma empresa ser nacional ou multinacional (SILVA NETTO et al., 2011). Considerando informações sobre a base de patentes, Suzigan et al. (2011) ressaltam que o Brasil até consegue avançar no número de patentes e nas interações entre áreas da ciência e setores da tecnologia, entretanto, isso apenas serve para que o gap tecnológico do país não aumente significativamente. ${ }^{1}$ Ribeiro et al. (2010) discutem a correlação entre produção científica e inovação tecnológica por meio do estudo de patentes, apontando para um padrão limitado e setorialmente localizado desse tipo de interação.

Dado esse quadro multifacetado, cabe destacar um esforço que busca aplicar a metodologia desenvolvida no Carnegie Mellon Survey e no Yale Survey para a análise daquelas interações ao caso brasileiro, por meio do denominado Survey Brasil (SUZIGAN et al., 2011). A partir das informações levantadas neste estudo, percebe-se que as instituições de ensino e pesquisa no Brasil convivem com uma característica dual. Por um lado, elas devem estar atentas a um crescimento cada vez maior do estoque de ciência desenvolvido nos países centrais; por outro lado, elas também devem se adaptar, em uma velocidade considerável, aos aumentos da demanda por serviços tecnológicos no país, contribuindo, desse modo, para o catching-up da estrutura produtiva nacional (SUZIGAN; ALBUQUERQUE, 2011). A partir da

1 Essa característica foi chamada de "efeito da rainha vermelha" por Ribeiro et al. (2010). 
base de dados gerada pelo Survey Brasil diversos estudos abordando temas específicos têm sido realizados. A atuação da universidade brasileira como solucionadora de problemas locais foi investigada por Costa et al. (2011) para o Rio Grande do Sul. Existem ainda estudos que observam se a proximidade geográfica influencia na interação das firmas com os grupos de pesquisa (GARCIA et al., 2011).

Em síntese, estas análises sugerem que as relações de cooperação tecnológica entre empresas inovadoras e outros agentes, incluindo universidades, têm uma relevância ainda restrita no Sistema Nacional de Inovação brasileiro, apesar do seu caráter multifacetado e do crescimento das mesmas no período recente. Há também diferenças significativas na propensão para cooperar em função da escala das empresas. De acordo com Melo e Rapini (2012), as grandes empresas nacionais (com mais de 500 empregados) mostram um esforço de cooperação próximo ao daquelas multinacionais presentes na economia brasileira e três vezes maiores do que as de portes menores.

Outro aspecto a ser considerado refere-se à forma como as empresas declaram inovar na Pintec, privilegiando a compra de equipamentos. Esse tipo de inovação gera poucos transbordamentos de tecnologias para as instituições de pesquisas, tendendo a privilegiar a aquisição de tecnologias já desenvolvidas para mercados distintos do brasileiro. Por esse mesmo motivo, observa-se que muitas empresas não constituem departamentos de $\mathrm{P} \& \mathrm{D}$ e deixam para utilizar os laboratórios das universidades quando necessário. Parte da explicação para isso pode estar na forte presença de empresas estrangeiras em setores dinâmicos da economia. Essas empresas preferem concentrar as atividades que necessitam de mais conhecimento científico em seus países de origem, deixando apenas questôes referentes à adaptação e inovações incrementais para serem feitas no Brasil (SUZIGAN, et al., 2011), o que reforça as conexôes com o exterior, em detrimento da infraestrutura local de C\&T. Ainda sobre o papel das empresas multinacionais, o trabalho de Silva Neto et al. (2011) tenta verificar a hipótese de que as multinacionais interagiriam mais com a infraestrutura de C\&T do que as firmas nacionais, dado que elas são muito interativas em seus países de origem. Entretanto, as evidências demonstram que isso não ocorre, indicando que as empresas que constituem filiais no Brasil passavam a se comportar da mesma forma daquelas aqui estabelecidas. Dentre as poucas diferenças, pode-se notar que as multinacionais buscam interações que conduzem à solução de um problema específico, enquanto as empresas nacionais atribuem maior importância para as atividades que geram aprendizado direto. Adicionalmente, observa-se que as multinacionais dariam maior importância à contratação de pesquisas junto 
a universidades e instituições de pesquisa, enquanto as nacionais atribuem maior relevância à transferência de tecnologia das universidades e à utilização de recursos disponíveis nestas instituições. Desse modo, não há indícios de que a cooperação entre empresas multinacionais e a infraestrutura de C\&T local seja mais «virtuosa» para o fortalecimento do Sistema Nacional de Inovação brasileiro, comparativamente à cooperação com aquelas de capital nacional. Na seção seguinte pretende-se contribuir para tal debate, a partir da utilização dos dados da Pintec, procurando-se mensurar a dimensão e a evolução, no tempo, dos relacionamentos cooperativos entre universidades e o setor produtivo no caso brasileiro, qualificando essa análise em função do porte e da origem de capital das empresas.

\section{Cooperação entre universidades e o setor produtivo no caso brasileiro segundo porte e origem do capital: evidências da Pintec}

\subsection{Fontes de dados}

A utilização de informações da Pintec para avaliar a importância da cooperação para a inovação tem sido objeto de diversas análises (CASSIOLATO; BRITTO; VARGAS, 2005; MARQUES; FREITAS; SILVA, 2007; KUPFER; AVELLAR, 2009; CUNICO et al., 2013; MACHADO; CABRAL; MATOS, 2015). Essas análises utilizam informações extraídas do questionário da Pintec, que capta a dimensão da cooperação do processo inovativo por meio de um conjunto de perguntas específicas. É importante salientar que, dentro da estrutura da Pintec, essas perguntas são respondidas pelas empresas que, de fato, implementaram inovações, o que delimita com mais clareza o objeto do estudo. Cabe salientar que a Pintec procura captar tanto a efetiva introdução de inovações (de produto ou processo) enquanto resultado de práticas cooperativas, como também o envolvimento efetivo das firmas em articulações cooperativas com diversos parceiros na realização de atividades inovativas, avaliando-se o tipo de parceiro, a importância atribuída a cada um deles, sua localização e o objeto da parceria.

$\mathrm{Na}$ análise desenvolvida a seguir, procurou-se articular informações sobre esforços inovativos desenvolvidos em cooperação com outras dimensões dos esforços e do desempenho inovativo das firmas. A análise contempla recortes por porte da empresa e origem do capital, obtidos a partir de tabulaçôes especiais da Pintec. No caso do porte, optou-se por um recorte amplo, que distingue empresas com mais ou menos de 500 empregados, enquanto no caso da origem de capital distinguem-se empresas 
de capital nacional e multinacional. $\mathrm{Na}$ análise realizada a seguir, foram consideradas informaçōes relativas a quatro períodos, caracterizados em função do ano de referência da pesquisa: 2003 (cobrindo o período 2001-2003), 2005 (referente a 2003-2005), 2008 (englobando o período 2006-2008) e 2011 (para o período 2009-2011). As tabulações especiais apresentadas foram geradas a partir de microdados da Pintec, disponibilizadas segundo os recortes mencionados.

As informaçōes que orientam a análise contemplam três aspectos principais. Em primeiro lugar, foram considerados dados gerais sobre as características e os esforços inovativos das firmas: total de empresas; empresas inovadoras; receita média de empresas segundo porte e origem do capital; taxa de inovação e esforços inovativos, incluindo o percentual de empresa inovadoras, o percentual de empresas com gastos em inovação, a intensidade de gastos em inovação como proporção da receita, o percentual de empresas com gastos em $P \& D$, a intensidade de gastos em $P \& D$ como proporção da receita e o percentual de gastos em $P \& D$ em relação ao total de gastos inovativos. Em segundo lugar, foram consideradas especificamente informações sobre inovação em cooperação: introdução de inovação em cooperação, em termos do percentual de empresas inovadoras, seja para inovação em produto, seja para inovação em processo; e realização efetiva de esforços inovativos em cooperação, distinguindo-se a taxa de cooperação total (em relação ao total de empresas), a taxa de cooperação em relação ao total de empresas inovadoras, o percentual de empresas inovadoras em cooperação com universidades e o percentual de empresas inovadoras em cooperação com centros tecnológicos. Por fim, foram consideradas informações sobre o apoio do governo a esforços inovativos, incluindo: percentual (taxa) de apoio em relação às empresas inovadoras; percentual das empresas inovadoras que tiveram acesso a algum tipo de incentivo fiscal à inovação; e percentual de empresas inovadoras que tiveram apoio governamental na forma de financiamentos a projetos em parceria voltados para atividades de pesquisa e desenvolvimento com universidades e institutos de pesquisa.

\subsection{Análise de resultados}

\subsubsection{Evolução geral de esforços inovativos}

A Tabela 1 apresenta resultados gerais relativos àqueles indicadores para o período 2003-2011, considerando recortes por porte e origem de capital. A taxa de inovação, medida pelo percentual de empresas inovadoras em relação ao total da amostra da Pintec, inicialmente oscilou numa faixa de 33\% entre 2003 e 2005, elevando-se para 


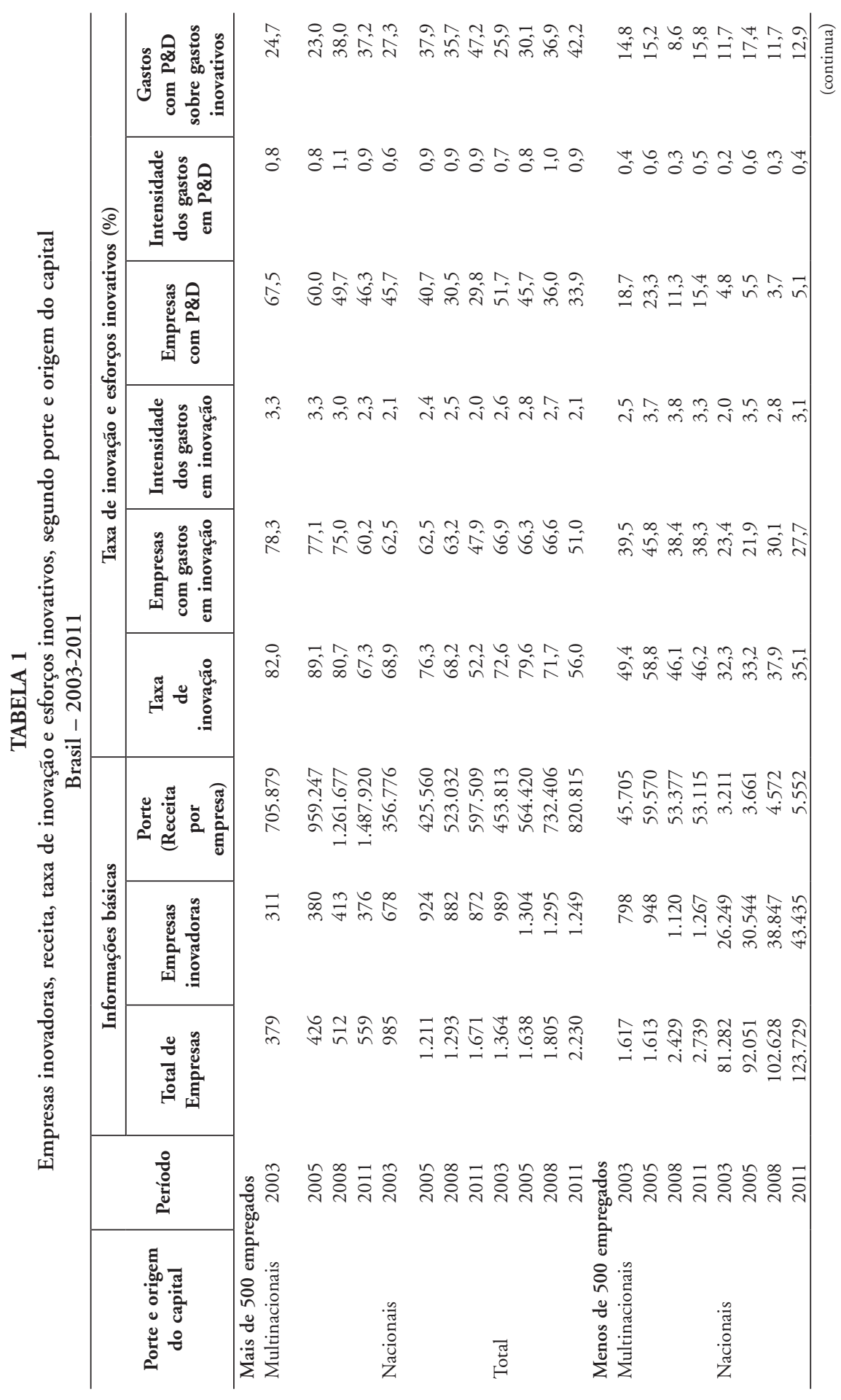




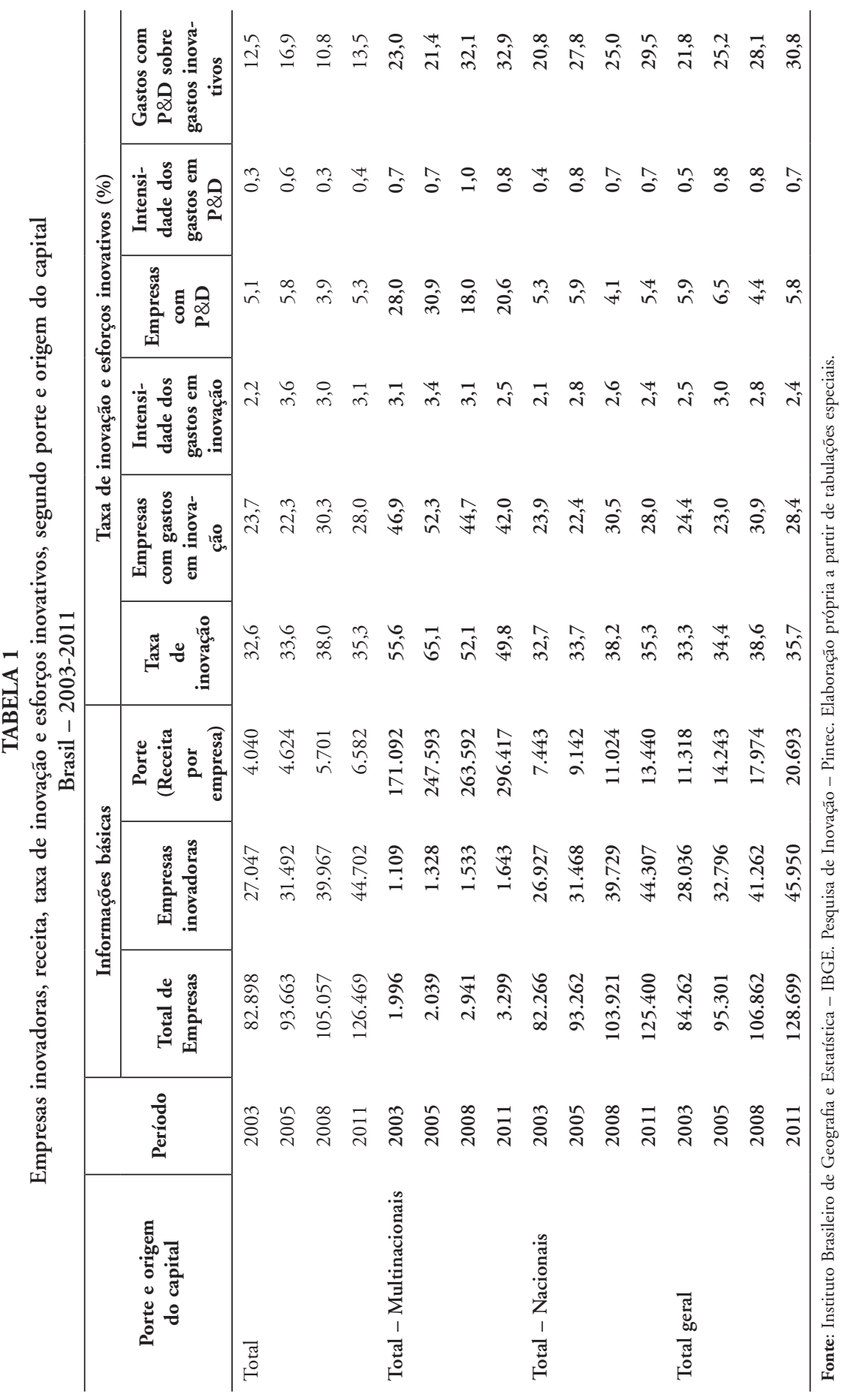


$38,7 \%$ na Pintec de 2008 e depois retornando para 35,7\% na de 2011. Ao longo desse período, o percentual de gastos inovativos em relação às vendas manteve-se entre $2,5 \%$ e $3,0 \%$. Já os esforços em P\&D foram realizados por apenas $5,8 \%$ das empresas em 2011, equivalendo a $0,7 \%$ das vendas naquele ano.

Essa análise geral pode ser mais bem qualificada quando se consideram recortes mais detalhados, por porte e origem do capital. A taxa de inovação apresenta-se mais elevada na faixa de mais de 500 empregados, na qual chegou a atingir 79,6\% em 2005, diminuindo para 56,0\% em 2011, devido, principalmente, à redução da taxa para empresas multinacionais de mais de 500 empregados, que decresceu de $89,1 \%$ em 2005 para $67,3 \%$ em 2011, enquanto para as nacionais do mesmo porte passou de $76,3 \%$ para $52,2 \%$,no período analisado, convergindo para valores próximos ao das multinacionais. Já na faixa de menos de 500 empregados, observa-se pequena evolução de 32,6\% em 2003 para 35,3\% em 2011, resultante principalmente do crescimento da taxa para empresas de capital nacional. Assim, em linhas gerais, verifica-se que a taxa de inovação evoluiu mais positivamente para as empresas de capital nacional, apesar de essas ainda apresentarem uma menor taxa geral quando comparadas com as multinacionais (35,3\% contra 49,8\% na comparação para 2011).

Ao longo do período investigado, as empresas com esforços inovativos ampliaram-se de 24,4\% em 2003 para 30,9\% em 2008, diminuindo para 28,4\% em 2011, movimento explicado pela trajetória mais positiva desse indicador para as empresas de menor porte (menos de 500 empregados) - com maior destaque para as de capital nacional - comparativamente à queda do percentual para a faixa de maior porte (mais de 500 empregados) - com redução mais acentuada para as multinacionais. No tocante à intensidade dos esforços inovativos (como percentual da receita), observa-se uma estagnação da taxa geral, que se manteve entre $2,5 \%$ e $3,0 \%$. Considerando um recorte por porte, quanto a esse indicador, identificam-se valores mais elevados para a faixa de menos de 500 empregados, em especial para as empresas de capital nacional, cujo percentual desses gastos em relação às vendas se elevou de 2,0\% em 2003 para 3,1\% em 2011. Como reflexo, observa-se uma aproximação geral dessa taxa entre empresas de capital nacional e multinacional.

No caso dos esforços em P\&D, estes eram realizados por apenas 5,8\% das empresas em 2011, apresentando valores mais elevados para as multinacionais (20,6\% das empresas em 2011). Ao longo do período investigado, esse percentual geral manteve-se estável na faixa de menos de 500 empregados (em torno de 5\% das empresas), mas reduziu-se expressivamente na faixa de mais de 500 empregados (de 51,7\% em 2003 para 33,9\% em 2011), tanto para as multinacionais como para 
as nacionais. A evolução da intensidade dos esforços em P\&D (como percentual da receita) também não foi expressiva, passando de $0,5 \%$ para $0,7 \%$ das vendas, entre 2003 e 2011. No caso das empresas de capital nacional, esse percentual aumentou de $0,4 \%$ para $0,7 \%$, no mesmo período, convergindo para valores próximos aos das multinacionais. Este indicador apresentava valores mais elevados na faixa de mais de 500 empregados - em torno de $0,9-1,0 \%$ das vendas - e, nessa faixa, não se identificam diferenciais significativos entre empresas nacionais e multinacionais. Apesar desse crescimento limitado dos gastos em P\&D, verifica-se um aumento da participação desses gastos em relação ao total dos gastos inovativos, que se ampliou de $21,8 \%$ em 2003 para 30,8\% em 2011. Um maior incremento foi observado para as empresas de capital nacional, de 20,8\% em 2003 para 29,5\% em 2011, e, dentre essas, para aquelas com mais de 500 empregados, cujo percentual evolui de $27,3 \%$ para $47,2 \%$, no mesmo período. Desse modo, há indícios de uma paulatina aproximação da intensidade dos esforços inovativos - incluindo os gastos em P\&D - realizados por empresas nacionais e multinacionais.

\subsubsection{Inovação em cooperação por porte e origem de capital}

As informaçôes contidas na Tabela 2 mostram que o percentual de empresas com atividades cooperativas para a inovação se ampliou de forma mais expressiva e consistente do que o da própria atividade inovativa, porém a partir de uma base inicial bastante limitada. De fato, as empresas inovativas que desenvolveram alguma atividade cooperativa para inovação passaram de 3,8\% em 2003 para 16,7\% em 2011, embora tal aumento tenha ocorrido a partir de um patamar inicial relativamente baixo. Também, a despeito da intensificação da inovação em cooperação do ponto de vista dos esforços, a introdução efetiva de inovações como resultado direto de práticas cooperativas é ainda restrita, apesar do avanço significativo obtido no período recente. No tocante à introdução de inovação em cooperação, observa-se que as empresas com inovação em produto ampliaram-se de $2,8 \%$ para $7,1 \%$ das empresas inovadoras, entre 2003 e 2011, enquanto para inovação em processo esse percentual evolui de $1,5 \%$ para $6,7 \%$, no mesmo período. No entanto, cabe ressaltar que o crescimento mais expressivo dessas taxas ocorreu, precisamente, para os grupos de empresas de capital nacional e daquelas de menor porte (tanto para inovação de produto como de processo), podendo-se, inclusive, verificar uma queda do percentual no caso da inovação de produto para empresas multinacionais de maior porte. 


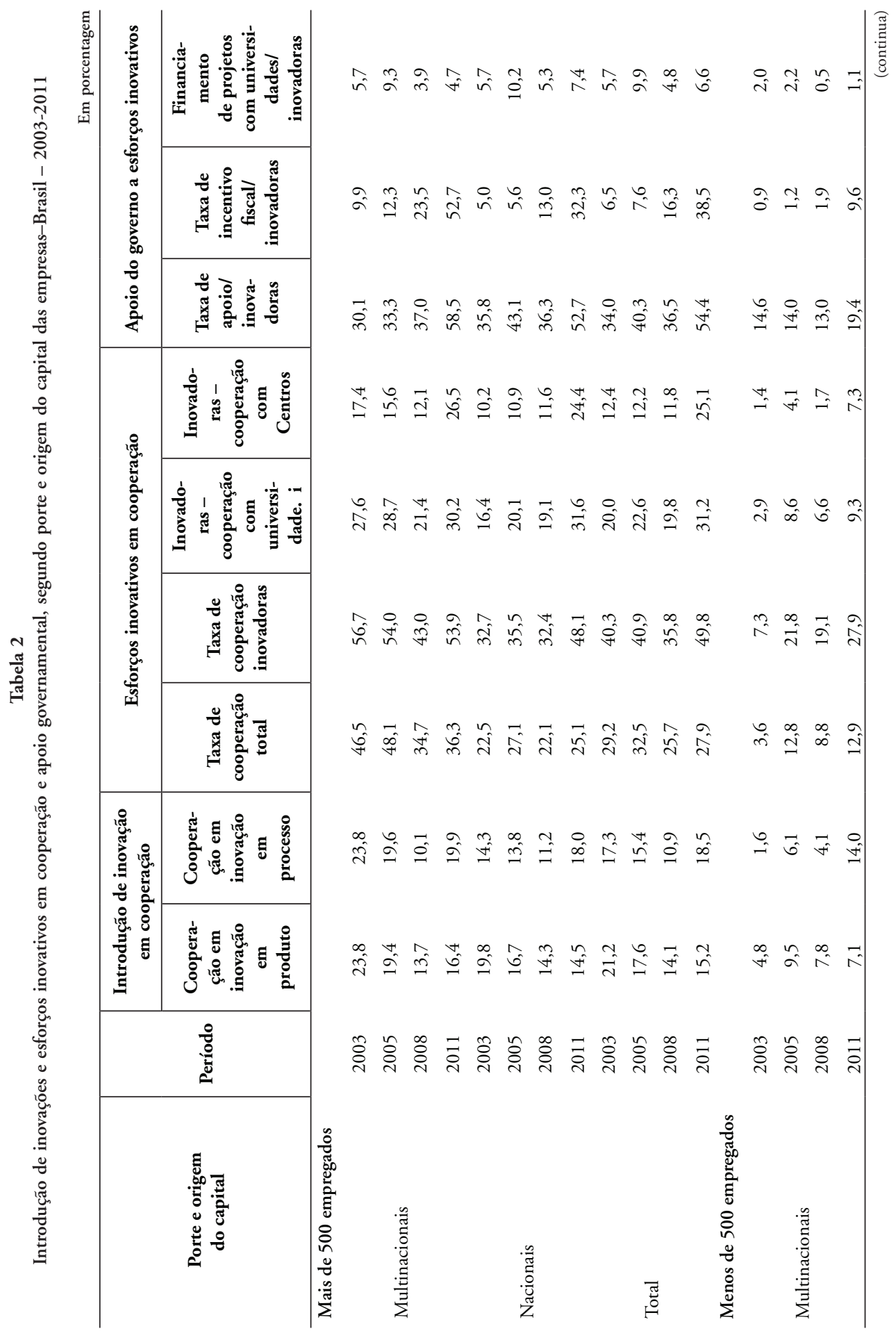




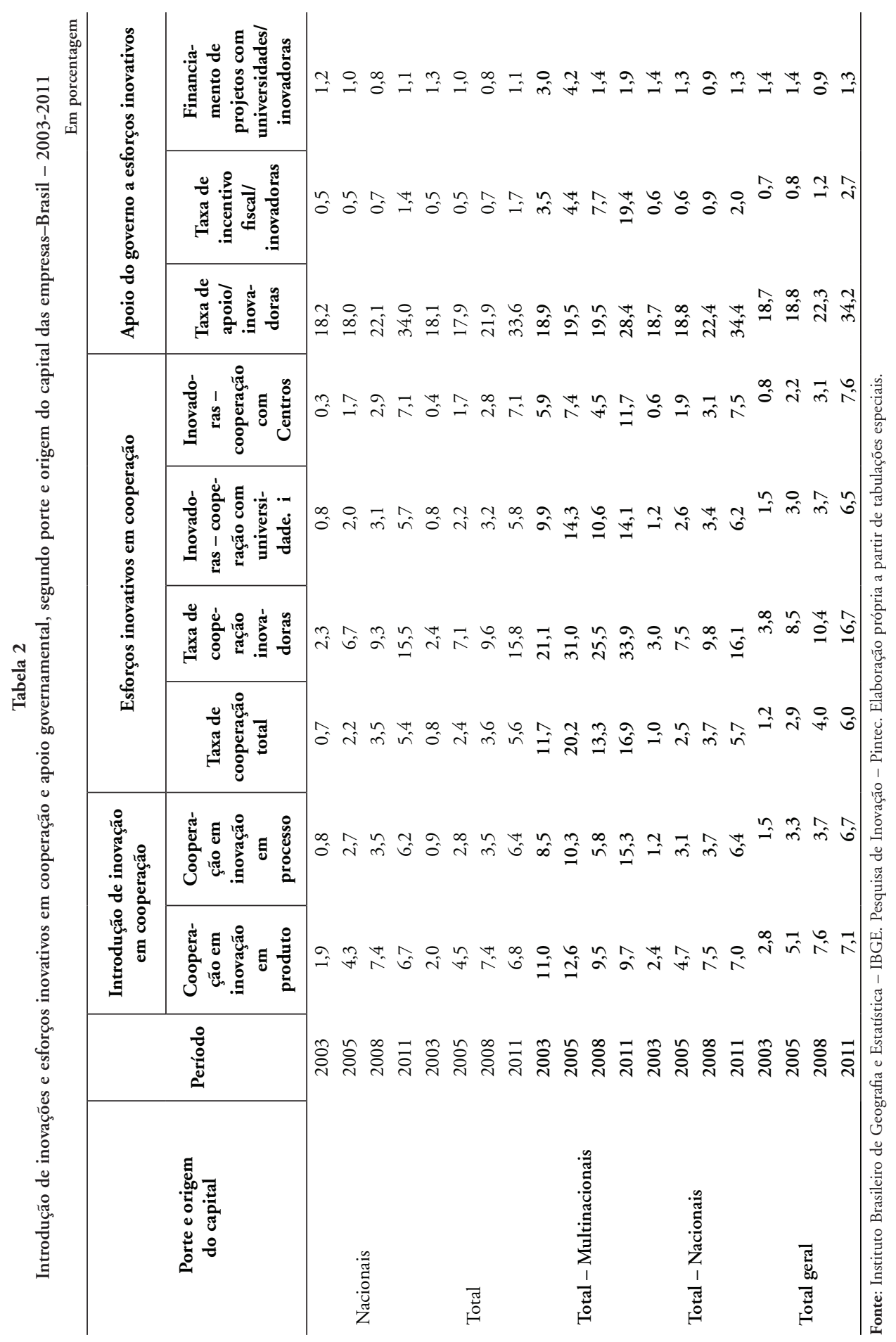


Com relação à taxa geral de cooperação, avaliada do ponto de vista dos esforços das firmas inovadoras e desagregada por ano, porte e origem de capital, observa-se um crescimento expressivo deste indicador, que evoluiu de 3,8\% do total de empresas inovadoras em 2003 para 16,7\% em 2011. Esse resultado é explicado, em boa parte, pelo aumento mais expressivo da taxa para o conjunto de empresas de capital nacional entre 2003 e 2011 (de 3,0\% para 16,1\% das empresas inovadoras) comparativamente às multinacionais (de 21,1\% para 33,9\% das empresas inovadoras). Considerando um recorte por porte, esse crescimento se explica, principalmente, pela faixa de menos de 500 empregados (elevação da taxa de 2,4\% para 15,8\% entre 2003 e 2011), em que a taxa para empresas de capital nacional se elevou de $2,3 \%$ para $15,5 \%$, contra uma evolução de $7,3 \%$ para $27,9 \%$ para as multinacionais. Quando se observa a faixa de mais de 500 empregados, verifica-se uma assimetria entre o crescimento da taxa para empresas de capital nacional e multinacionais. De fato, enquanto para as primeiras verifica-se um incremento na taxa, que passou de $32,7 \%$ das empresas inovadoras para $48,1 \%$, para as multinacionais ocorreu decréscimo de 56,7\% para 53,9\%. Como consequência, a taxa geral naquela faixa de porte se elevou de $40,3 \%$ para $49,8 \%$, impulsionada pelo crescimento das empresas de capital nacional de maior porte.

Entretanto, os dados que mais chamam a atenção referem-se a um ponto específico de interesse deste artigo: a participação de universidades em arranjos cooperativos. A Tabela 2 também apresenta informações sobre a evolução intertemporal da taxa de cooperação com universidades e institutos de pesquisa e com centros de capacitação profissional e assistência técnica, desagregadas por porte e origem de capital para os vários anos considerados (2002, 2005, 2008 e 2010). No caso da taxa de cooperação com universidades e institutos de pesquisa, desagregada por ano, porte e origem de capital, também se observa um crescimento expressivo de $1,5 \%$ para $6,5 \%$, entre 2003 e 2011. Essa evolução também é explicada, em boa parte, pelo aumento mais significativo da taxa para o conjunto de empresas de capital nacional no período 2003-2011 (de 1,2\% para 6,2\%), comparativamente às multinacionais (de $9,9 \%$ para $14,1 \%$ ). Considerando um recorte por porte, esse crescimento foi mais intenso na faixa de menos de 500 empregados, na qual a taxa para empresas de capital nacional se elevou de 0,8\% para 5,7\% entre 2003 e 2011, contra uma evolução de $2,9 \%$ para $9,3 \%$ no caso das multinacionais. Para a faixa de mais de 500 empregados, no mesmo período, esta taxa ampliou-se de 16,4\% para $31,6 \%$, para as empresas de capital nacional, e de $27,6 \%$ para $30,2 \%$, no caso das multinacionais. Como consequência, a taxa geral nesta faixa de porte se elevou 
de 20,0\% em 2003 para 31,2\% em 2011, impulsionada, principalmente, pelas empresas de capital nacional.

No tocante à cooperação com centros de capacitação profissional e assistência técnica, desagregada por ano, porte e origem do capital, também se observa um crescimento expressivo desta taxa, que passou de $0,8 \%$ das empresas inovadoras em 2003 para 7,6\% em 2011. Tal crescimento, inclusive, é superior ao observado no caso da cooperação com universidades, no período considerado. Esse comportamento também é explicado em função do crescimento mais expressivo da taxa para o conjunto de empresas de capital nacional entre 2003 e 2011 (de 0,6\% para $7,5 \%$ ) comparativamente às empresas multinacionais (de 5,9\% para $11,7 \%$ das empresas inovadoras). Segundo o recorte por porte, tal desempenho é explicado, principalmente, pela faixa de menos de 500 empregados, na qual a taxa para empresas de capital nacional se elevou de $0,3 \%$ para 7,1\%, entre 2003 e 2011, contra uma evolução de $1,4 \%$ para $7,3 \%$ para as multinacionais. $\mathrm{Na}$ faixa de mais de 500 empregados, também se verifica um maior crescimento da taxa entre as empresas de capital nacional (de 10,2\% para 24,4\%) comparativamente às multinacionais (de $14,3 \%$ para $17,8 \%$ ). Como consequência, a taxa geral nesta faixa de porte se elevou de $17,4 \%$ para $26,5 \%$. Assim, constata-se, no caso da cooperação com centros de capacitação profissional e assistência técnica, um aumento generalizado, incluindo diferentes portes e origens de capital, apesar do mesmo ter sido mais intenso para empresas de capital nacional e de menor porte.

Uma observação interessante diz respeito à diferença de comportamento entre as empresas nacionais e estrangeiras. Considerando o recorte por porte, verifica-se que as empresas estrangeiras registram maior percentual de arranjos cooperativos. No entanto, as evidências também apontam que diferença entre as grandes empresas nacionais e estrangeiras é menos pronunciada e que este diferencial se reduziu expressivamente ao longo do período considerado. De fato, considerando em conjunto os indicadores definidos pela taxa geral de cooperação, pelo percentual de cooperação com universidade e pelo percentual de cooperação com centros de capacitação profissional e assistência técnica, verifica-se que, na média, a relação entre os valores observados para esses indicadores para empresas multinacionais e nacionais decresceu de aproximadamente oito vezes em 2003 para duas vezes em 2011. Na faixa de mais de 500 empregados, esses indicadores eram praticamente equivalentes para os dois grupos de empresas em 2011. Além disso, no caso específico das empresas nacionais, o tamanho "importa" no sentido de induzir a intensificação de práticas cooperativas, pois, apesar da expressiva redução desses diferenciais 


\subsubsection{Apoio governamental à inovação em cooperação}

Outro aspecto a ser analisado refere-se ao papel do apoio governamental no sentido da criação de estímulos à intensificação de esforços inovativos e, em especial, daqueles esforços que envolvem práticas cooperativas com a infraestrutura de C\&T. Com esse intuito, é possível considerar informações sobre o apoio governamental à intensificação desses esforços fornecidas pela Pintec, desagregadas por porte e origem do capital. ${ }^{2}$ Os dados da Tabela 2 apontam para um crescimento das empresas inovativas que relatavam algum tipo de apoio governamental nessa direção, cujo percentual elevou-se 18,7\% em 2003 para 34,2\% em 2011. Esse aumento foi mais expressivo no caso das empresas de capital nacional, cujo percentual passou de $18,7 \%$ para $34,4 \%$, nesse período, enquanto para as multinacionais a taxa elevou-se de $18,9 \%$ para 28,4\%. Quanto a esse aspecto, diferenças importantes em termos do porte das empresas inovadoras podem ser mencionadas. No caso daquelas de maior porte, observa-se um crescimento desse percentual de 34,0\% em 2003 para 54,4\% em 2011. O aumento ocorreu tanto para empresas inovadoras de capital nacional de maior porte (de 35,8\% para 52,7\%) quanto para as multinacionais de maior porte (de 30,1\% para 58,5\%). Já na faixa de menos de 500 empregados, apesar do menor acesso a esse tipo de apoio, o crescimento foi bastante significativo, evoluindo de $18,1 \%$ para $33,6 \%$, no mesmo período, impulsionado principalmente pela ampliação nas empresas de capital nacional (de 18,2\% para 34,0\%). Deve-se ressaltar que existem dificuldades naturais para o acesso das empresas de menor porte ao apoio governamental, tais como a oferta de garantias exigidas pelas agências de fomento, dificuldades gerenciais inerentes a este tipo de empresa e as próprias limitaçóes dos programas de subvenção econômica adaptados às suas características.

A partir das informaçôes da Pintec, é possível considerar também a intensidade da utilização de incentivos fiscais por empresas inovadoras. Nesse sentido, os dados disponíveis indicam que a utilização desses instrumentos é ainda limitada, apesar de ter se elevado significativamente no período mais recente, evoluindo de $0,7 \%$ para 2,7\%, entre 2003 e 2011, destacando-se o maior crescimento desse percentual para empresas de capital multinacional (de 3,5\% para 19,4\%), comparativamente às de capital nacional (de 0,6\% para 2,0\%). Em termos das faixas de porte, o uso desse instrumento era claramente maior para as empresas de porte mais elevado, atingindo $38,5 \%$ para o conjunto de empresas dessa faixa, elevando-se para 52,7\%

2 Deve-se ressaltar que as próprias universidades e centros de pesquisa também passaram a buscar apoio financeiro do governo por meio de programas como a Lei de Inovação e dos Fundos Setoriais. 
no caso das multinacionais, contra $32,3 \%$ para as nacionais. Em contraste, na faixa de menos de 500 empregados, esse percentual limitava-se a 1,7\% para o conjunto das empresas em 2011, atingindo 1,4\% no caso daquelas de capital nacional e 9,6\% para as multinacionais. Assim, é possível constatar que o apoio governamental, incluindo incentivos fiscais a esforços inovativos, não tem sido capaz de reduzir as desigualdades em termos de porte e origem de capital que caracterizam o padrão de realização desses esforços no caso brasileiro, sendo mais facilmente acessado por empresas de maior porte e/ou de capital multinacional.

No tocante, especificamente, aos esforços inovativos realizados em cooperação com universidades, é possível considerar a evolução do percentual de empresas inovadoras que obtiveram financiamento para projetos de pesquisa e desenvolvimento em parceria com universidades e institutos de pesquisa, a partir das informações da Pintec. Nesse caso, observa-se que a cobertura deste apoio é ainda bastante restrita. De fato, para o conjunto das empresas inovadoras, esse percentual apresenta-se praticamente estagnado, passando de 1,4\% em 2003 para 1,3\% em 2011. Para o conjunto de empresas nacionais, esse percentual limitava-se a 1,3\% das firmas inovadoras em 2011, enquanto para as multinacionais correspondia a 1,9\%. Em termos do porte das empresas, esse tipo de apoio era mais efetivo para aquelas de maior porte (mais de 500 empregados), atingindo 6,6\% das empresas inovadoras em 2011, contra apenas 1,1\% para de menor porte (menos de 500 empregados) naquele ano. Entre as empresas de maior porte, apesar do percentual restrito desse tipo de apoio, o mesmo apresentava-se, em 2011, mais elevado para as nacionais $(7,4 \%)$ do que para as multinacionais $(4,7 \%)$. Esses valores, além de reduzidos, revelando a baixa cobertura do apoio governamental ao financiamento da cooperação universidade-indústria, apresentam grande instabilidade ao longo do período considerado, sugerindo que esse tipo de apoio não se encontra plenamente incorporado ao arcabouço de instrumentos de apoio definidos no âmbito das políticas de inovação.

\section{Considerações finais}

O presente artigo procurou tratar, de forma original, os dados contidos nas Pintec, tendo como foco as relações de cooperação para inovação entre empresas inovadoras e universidades, institutos de pesquisa e centros de capacitação profissional e assistência técnica, considerando recortes por porte e origem do capital das empresas inovadoras. Apesar de a análise realizada não ter incorporado um recorte setorial, há indícios de que a intensidade daquelas práticas tende a variar em função das especificidades 
setoriais em termos do mix particular dos atributos de porte e origem de capital prevalecentes em cada contexto, das características da base de conhecimento e da dinâmica concorrencial e de estímulos de política particulares que afetam cada setor. ${ }^{3}$

Em linhas gerais, os indicadores apresentados apontam para um quadro positivo no tocante à evolução das relações de cooperação entre empresas e instituições geradoras de conhecimento científico e tecnológico no final da década de 2000. Logo, seguindo a literatura, deveríamos estar observando um avanço da atividade inovativa que se traduziria em efeitos positivos no desempenho da indústria brasileira. Apesar de se esperar que essa dinâmica tenha um impacto positivo sobre produção e produtividade das firmas, o caráter predominantemente incremental das inovações geradas pode não ser capaz de gerar ganhos substantivos nestas variáveis. Se, por um lado, os resultados sinalizam impactos positivos, por outro, é forçoso reconhecer que este processo se dá numa conjuntura de forte desaceleração da atividade econômica, como um todo, e da indústria em particular. Assim, os efeitos positivos de atividade inovativa podem ser compensados no agregado com os efeitos pró-cíclicos, em termos da produtividade, resultantes da queda da atividade econômica.

A tendência geral observada reforça evidências apresentadas pelas análises de outros autores. As empresas inovadoras com relações de cooperação têm ampliado sua participação no total de empresas inovadoras; no entanto, observa-se que as nacionais registram uma proporção menor de arranjos colaborativos, apesar de, em termos evolutivos, vislumbrar-se uma tendência de aproximação com os índices das empresas multinacionais. Verificou-se, também, um crescimento mais intenso das relações de cooperação com centros de capacitação, sugerindo, no agregado, uma efetividade das políticas de inovação que estimulam a expansão de tais instituições. A constituição de uma rede de centros de prestação de serviços tecnológicos especializados por meio da organização do sistema Sibratec parece ter contribuído nessa direção, e a expectativa é que o processo se intensifique com a criação da Embrapii.

Finalmente, deve-se reconhecer que, a despeito das melhorias observadas em indicadores examinados, esses avanços ainda não se traduzem de forma mais sistemática no desempenho do conjunto da indústria brasileira. Certamente, há que se levar em conta que alguns fatores macroeconômicos nacionais, combinados com outros relacionados a transformaçōes na estrutura produtiva internacional, têm forte relevância no desempenho recente da indústria brasileira. Problemas em

3 A análise de Britto e Vargas (2015), por exemplo, aponta para uma maior intensidade das articulaçôes cooperativas entre o setor empresarial e a infraestrutura de C\&T no caso do setor farmacêutico, que tem sido objeto de um conjunto mais concertado de estímulos de política. 
variáveis como câmbio, juros domésticos e na adequação do nível do investimento privado e da infraestrutura pública podem desempenhar um papel relevante nos primeiros, enquanto, no tocante às transformaçōes da estrutura produtiva no plano internacional, destacam-se o avanço da China como fábrica do mundo, a formação de cadeias internacionais de valor e a velocidade das inovaçôes tecnológica recentes. Entretanto, levando-se em conta a perceptível melhoria dos dados que apontam para intensificação de práticas cooperativas na última Pintec (2011), há evidências de que essa tendência representa um vetor positivo em relação às perspectivas do desenvolvimento da indústria brasileira, podendo vir a contribuir para a intensificação de esforços inovativos realizados pelo setor empresarial, com possíveis desdobramentos sobre a "qualidade" dos investimentos e sobre a produtividade da indústria.

\section{Referências bibliográficas}

ALBUQUERQUE, E. M.; SILVA, L. A.; PÓVOA, L. Diferenciação intersetorial na interação entre empresas e universidades no Brasil. São Paulo em Perspectiva, v. 19, n. 1, p. 95-104, 2005.

ALBUQUERQUE, E. M.; SILVA, L.; RAPINI, M.; SOUZA, S. Interactions between firms and universities in on immature system of innovation: a survey of $R \& D$ performer firms in Minas Gerais, Brazil. In: III GLOBELICS CONFERENCE Pretoria, South Africa, 2005. Disponível em: <http://www.globelics2005africa.org.za/papers/p0050/index.php>.

ALBUQUERQUE, E. M.; SUZIGAN, W.; CARIO, S.; FERNANDES, A.; SHIMA, W.; BRITTO, J.; BARCELOS, A.; RAPINI, M. An investigation on the contribution of universities and research institutes for maturing the Brazilian innnovation system: preliminary results. In: IV GLOBELICS CONFERENCE. Mexico City, 22-24 September 2008.

ALBUQUERQUE, E. M.; SUZIGAN, W.; KRUSS, G.; LEE, K. (Ed.). Developing National Systems of Innovation - university-industry interactions in the global south. [S.1.]: Edward Elgar Publishing, 2015.

ARCHIBUGI, D.; IAMMARINO, S. The globalization of technological innovation: definition and evidence. Review of International Political Economy, v. 9, n. 1, p. 98-122, 2002.

AROCENA, R.; SUTZ, J. Knowledge, innovation and learning: system and policies in the north and in the south. In: CASSIOLATO, J. et al. (Org.). System of innovation and development - Evidences from Brazil. Northampton, MA: Edward Elgar, 2003.

ARZA, V. Chanel, benefits and risks of public-private interactions for knowledge transfer: a conceptual framework inspired by Latin America. Science and Public Police, v.37, n. 7, p. 499-511, 2010. 
AZEVEDO, P.; CARIO, S. A. F.; MELO, P. A. As relaçôes universidade e empresa para o desenvolvimento inovativo sob nova perspectiva: a abordagem institucionalista-evolucionária. In: V COLÓQUIO INTERNACIONAL DE GESTÃO UNIVERSITÁRIA - CIGU. Mar del Plata - Argentina, 2, 3 e 4 de dezembro de 2015.

BEKKERS, R.; FREITAS, I. Analysing knowledge transfer channels between universities and industry: to what degree do sectors also matter? Research Policy, v. 37, n. 10, p,18381553, 2008.

BERNARDES, A. T.; ALBUQUERQUE, E da M. Cross-over, thresholds, and interactions between science and technology: lessons for less-developed countries, Research Policy, v. 32, p. 865-885, 2003.

BONACCORSI, A.; PICCALUGA, A. A. Theoretical framework for the evaluation of university-industry relationships. $R \& D$ Management, v. 24, n. 3, p. 229-240, 1994.

BRASCOMB, L. M.; KODAMA, F.; FLORIDA, R. Industrializing knowledge - universityindustry linkages in Japan and the United States. Cambridge-Londres: The MIT Press, 1999.

BRITO, J.; DEL-VECCHHIO, R. Patterns of university-industry interactions in Brazil: an explanatory analysis using the instrumental of graph theory. Quality \& Quantity, v. 48, n. 4, p. 1867-1892, July 2014.

BRITTO, J.; OLIVEIRA, B. F. Padrões setoriais de interação universidade-empresa no Brasil: um mapeamento de competências a partir de informaçôes da pesquisa "Brazil Survey". Revista de Economia, v. 37, número especial, p. 167-212, 2011.

BRITTO, J.; VARGAS, M. A. A systemic innovation policy with an inclusive perspective: the evolution of the Brazilian policy to the pharmaceutical sector. [S.1.], 2015 (Globelics Working Paper Series, n. 2015-07).

BRITTO, J. N. P.; STALLIVIERI, F. Innovation Policy in Brazil at a Crossroads: Institutional Hysteresis and the Need of Coordination., In: IX GLOBELICS INTERNATIONAL CONFERENCE. Buenos Aires, November 15-17 2011.

CASSIOLATO, J. E.; BRITTO, J.; VARGAS, M. A. Arranjos cooperativos e inovação na indústria brasileira. In: DE NEGRI, J. A.; SALERNO, M. S. (Org.). Inovaçôes, padrôes tecnológicos e desempenho das firmas industriais brasileiras. Brasília: Ipea, 2005. p. 511-576.

COHEN, W. et al. Links and impacts: the influence of public R\&D on industrial research. Management Science, v. 48, n. 1, p. 1-23, 2002.

COSTA, A.; RUFFONI, J.; PUFFAL, D. Proximidade geográfica e interação universidade-empresa no Rio Grande do Sul. Revista de Economia, v. 37, n. 3, p. 213-238, 2011. 
CUNICO, E.; CIRANI, C. B. S.; TEIXEIRA, C. E.; FREITAS, W. R. S. A estrutura do SNI brasileiro e a cooperação tecnológica como instrumento para geração de inovação na indústria nacional. In: XVI SEMEAD, SEMINÁRIOS EM ADMINISTRAÇÃO. Anais... São Paulo: FEA-USP, 2013.

DAGNINO, R. A relação universidade-empresa no Brasil e o "argumento da hélice tripla". Convergencía Revista de Ciencias Sociales, v. 11, n. 35, p. 253-291, 2004.

DUTRÉNIT, G.; ARZA, V. Channels and benefits of interactions between public research organisations and industry: comparing four Latin American countries. Science and Public Policy, v. 37, n. 7, p. 473-484, 2010.

ETZKOWITZ, H. Hélice tríplice: universidade-industria-governo-inovação em movimento. 1. ed. Porto Alegre: EdiPUCRS, 2013.

ETZKOWITZ, H.; LEYDERSDORFF, L. The dynamics of innovation: from national systems "mode 2" to a tripe helix of university-industry-government relations. Research Policy, v. 29, n. 2, p. 109-123, 2000.

FERNANDES, A. C.; CAMPELO DE SOUZA, B.; STANFORD SILVA, A.; SUZIGAN, W.; CHAVES, C. V.; ALBUQUERQUE, E, M. Academy-industry links in Brazil: evidence about channels and benefits for firms and researchers. Science and Public Policy, v. 37, n. 7, p. 485-498, 2010.

FUENTES, C.; DUTRÉNIT, G. Best channels of academia-industry interaction for longterm benefit. Research Policy, v. 41, n. 9, p. 1666-1682, 2012.

GARCIA, R.; ARAÚJO, V. C.; MASCARINI, S.; SANTOS, E.G. Os efeitos da proximidade geográfica para o estímulo da interação universidade-empresa. Revista de Economia, v. 37, n. 3, p. 307-330,2011.

GONÇALO, C.; ZANLUCHI, J. B. Relacionamento entre empresa e universidade: uma análise das características de cooperação em um setor intensivo em conhecimento. Base (UNISINOS), v. 8, n. 3, p. 261-272, 2011.

KLEVORICK, A.; LEVIN, R.; NELSON, R.; WINTER, S On the sources and significance of interindustry differences in technological opportunities. Research Policy, v. 24, n. 2, p.185-205, 1995.

KUPFER, D.; AVELLAR, A. P. Innovation and cooperation: evidences from the Brazilian innovation survey. In: XXXVII ENCONTRO NACIONAL DE ECONOMIA. Anais... Foz do Iguaçu: Anpec, 2009.LUNDVALL, B. A. Introduction. In: LUNDVALL, B. A. National Systems of Innovation: towards a Theory of Innovation and Interactive Learning. London: Printer Publishers, 1992. 
The university in the learning economy. [S.l.], 2002 (DRUID Working Paper n. 02-06).

MACHADO, D. Q.; CABRAL, J. E. O.; MATOS, F. R. N. Padrões na utilização de agentes de cooperação para a inovação na indústria de transformação brasileira. Brazilian Business Review, v. 12, n. 1, p. 105-128, jan.-fev. 2015.

MARQUES, R. A.; FREITAS, I. M. B.; SILVA, E. M. P. Colaboração com universidade e as atividades para inovação de empresas brasileiras. Engevista, v. 9, n. 2, p. 112-128, dezembro 2007.

MAZZOLENI, R. Catching up and academic institutions: a comparative study of past national experiences. Journal of Development Studies, v. 44, n. 5, p. 678-700, 2008.

MAZZOLENI, R.; NELSON, R. Public research institutions and economic catch-up. Research Policy, v. 36. p. 1512-1528, 2007.

MELO, L. M.; RAPINI, M. Financing innovation in Brazil: empirical evidence and implicit S\&T. Belo Horizonte: UFMG/Cedeplar, 2012.

MOWERY, D.; NELSON, R.; SAMPAT, B.; ZIEDONIS, A. Ivory tower and industrial innovation: university-industry technology transfer before and after the Bayh-Dole Act. Stanford: Stanford University, 2004.

NARIN et al. The increase linkage between U.S. technology and public science. Research Policy,v 26, p. 317-330, 1997.

NOVELI, M.; SEGATTO, A. P. Processo de cooperação universidade-empresa para a inovação tecnológica em um parque tecnológico: evidências empíricas e proposição de um modelo conceitual. Revista de Administração e Inovação, v. 9, n. 1, p. 81-105, 2012.

PARANHOS, J.; HASENCLEVER, L. Industry-university interactions in the pharmaceutical system of innovation: Brazilian and international data. In: DRUID-DIME ACADEMY WINTER PhD CONFERENCE. Aalborg: DRUID-DIME, 2009.

PERKMANN, M.; WALSH, K. Engaging the scholar: three types of academic consulting and their impact on university and industry. Research Policy, v 37, n. 10, p. 1884-1891, 2008.

PINHO, M. A visão das empresas sobre a relação entre universidade e empresas no Brasil: uma análise baseada nas categorias de intensidade tecnológica. Revista de Economia, v. 37 , n. 3, p. 279-306, 2011.

PINHO, M.; TORKOMIAN, A. L.V.; SANTOS, M. E. R. As relaçôes entre universidades e empresas no Brasil: mais do que se supõe, menos do que se precisa. In: XIV CONGRESSO IBERO-LATINOAMERICANO DE GESTÃO DA TECNOLOGIA - ALTEC 2015. Porto Alegre, outubro 2015. 
PÓVOA, L.; MONSUETO, S. Tamanho das empresas, interação com as universidades e inovação. Revista de Economia, v. 37, n. 3, p. 7-21, 2011.

RAPINI, M.; CASSIOLATO, J. E.; BITTENCOURT P. A relação universidade-indústria no Sistema Nacional de Inovação brasileiro: uma síntese do debate e perspectivas recentes. Rio de Janeiro: IE-UFRJ, 2007 (BRICS Projetc, Research Paper 11/07).

RAPINI, M.; CHIARINI, T.; BITTENCOURT, P. F. Innovation system and development in Latin America: university-industry interactions in Brazil. In: XVIII ENCONTRO NACIONAL DE ECONOMIA POLÍTICA. Anais... Belo Horizonte: Sociedade Brasileira de Economia Política, 2013.

RAPINI, M. Interação universidade-empresa no Brasil: evidência do diretório dos grupos do CNPq. Estudos Econômicos, v 37, n. 1, p. 211-233, 2007.

RIBEIRO, L.; RUIZ, R. M.; BERNARDES, A. T.; ALBUQUERQUE, E. M. Matrices of science and technology interactions and patterns of structured growth: implications for development. Scientometrics, v 83, n. 1, p. 55-75, 2010.

RIGHI, H. M. O panorama da interação entre universidade e indústria no Brasil. Dissertação (Mestrado) - Universidade Estadual de Campinas, Campinas, 2009.RIGHI, H. M.; RAPINI, M. S. Interação universidade-empresa no Brasil em 2002 e 2004: uma aproximação a partir dos grupos de pesquisa do CNPq. Revista Economia, v. 8, n. 2, p. 248-268, 2007.

ROSENBERG, N. Inside the balck box: technology and economics. Cambridge: Cambridge University, 1982.

SCHARTINGER, D.; RAMMER, C.; FRÖHLICH, J. Knowledge interactions between universities and industry in Austria: sectorial patterns and determinants. Research Policy, v. 31, n. 3, p.303-328, Mar. 2002.

SEGATTO, A. P. M.; SBRAGIA, R. O processo de cooperação universidade-empresa em universidades brasileiras. Revista de Administração, v. 37, n. 4, p. 58-71, out./dez. 2002.

SILVA NETO, F; SANTOS, U. P.; OLIVEIRA, V. P.; CASTRO, P. G.; FRANCO, L. T. M.; NEGRI, F. A interação universidade/instituto público de pesquisa e empresa no Brasil: resultados comparativos entre o relacionamento com empresas nacionais e multinacionais. Revista de Economia, v. 37, n. 3, p. 117-140, 2011.

SILVA, L. A. Padrôes de interação entre ciência e tecnologia: uma investigação a partir de estatísticas de artigos e patentes. Dissertação (Mestrado) - Universidade Federal de Minas Gerais, Centro de Desenvolvimento e Planejamento Regional, Belo Horizonte, 2003. 
SUZIGAN, W.; ALBUQUERQUE, E.; CARIO, S. Em busca da inovação: interação universidade-empresa no Brasil. Belo Horizonte: Editora Autêntica, 2011.

SUZIGAN, W.; ALBUQUERQUE, E. A interação entre universidades e empresas em perspectiva histórica no Brasil. Belo Horizonte: UFMG/Cedeplar, 2008 (Texto para discussão n. 329). The underestimated role of universities for the Brazilian system of innovation. Revista de Economia Política, v. 31, n. 1, p. 3-30, 2011.

SUZIGAN, W.; RAPINI, M.; ALBUQUERQUE, E. A changing role for universities in the periphery. Belo Horizonte: Cedeplar-UFMG, 2011 (Textos para discussão, 420).

TETHER, B. S. Who co-operates for innovation, and why. An empirical analysis. Research Policy, v. 31, n. 6, p. 947-967, 2002.

TIJSSEN, R. Universities and industrially relevant science: toward mensurement models and indicators of enterpreneurial orientation. Research Policy, v. 35, n. 10, p. 1569-1585, 2006. VELHO, L.; SAENZ, T. W. R\&D in the public and private sector in Brazil: complements or substitutes? Maastricht, The Netherland: UNU/INTECH, 2002 (UNU/INTECH Discussion Paper, 2002-8). 